\title{
ENERGY DEPENDENCE OF COLLECTIVE FLOW OF NEUTRONS AND CHARGED PARTICLES IN ${ }^{197} \mathrm{AU}+{ }^{197} \mathrm{AU}$ COLIISIONS
}

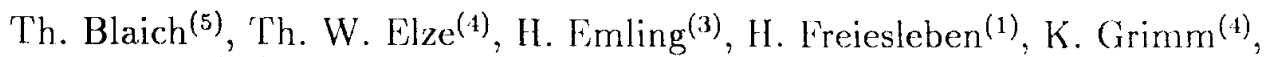
W. Henning ${ }^{(3,7)}$, R. Holzmann ${ }^{(3)}$, J. G. Keller ${ }^{(1)}$, H. Klingler ${ }^{(4)}$, J. V. Kratz ${ }^{(5)}$, R. Kulessa ${ }^{(2)}$, D. Lambrecht ${ }^{(5)}$, S. Lange ${ }^{(1)}$, Y. Leifels ${ }^{(1)}$, E. Lubkiewicz ${ }^{(2)}$, E. F. Moore ${ }^{(3,6)}$, W. Prokopowicz ${ }^{(2)}$, R. S hmidt ${ }^{(3)}$. C. Schütter ${ }^{(4)}$, II. Spies ${ }^{(4)}$, K. Stelzer ${ }^{(4)}$, I. Stroth ${ }^{(3)}$, E. Wajda ${ }^{(2)}$, W. Walus ${ }^{(2)}$, M. Zinser ${ }^{(3)}$, E. Zude ${ }^{(3)}$ and the FOPI-collaboration ${ }^{(+)}$

(1) Institut für lexperimentalphysik, Ruhr-Universität Buchum. 14801 Bochum, Germany

(2) Institute of Physics, Jagellonian University, PL-30-059 Cracow, Poland

(3) Gesellschaft für Schwerionenforschung, 6.1291 Darmstadt, Germany

(1) Institut für Kermphysik, Johann-Wolfgang-Goethe-Lniversität, 60325 Frankfurt, Germany

(5) Institut für Kernchemie, Johannes-Gutenberg-Uriversität, 55099 Mainz, Germany

(6) present address: Dept. of Physics, North Carolina State University, P.O.Box 8202, Raleigh, NC 27695-8202

(7) present address: Physics Division, Argonne National Laboratory, Argonne, IL 60439

\section{INTRODUCTION}

Collective flow of nuclear matter is one important aspect of the research performed at heavy ion accelerator laboratories. The phenomenon was predicted on the basis of hydrodynamical calculations [1], and experimemal evidence was first presented for the systems ${ }^{93} \mathrm{Nb}+{ }^{93} \mathrm{Nb}$ and ${ }^{197} \mathrm{Au}+{ }^{197} \mathrm{Au}$ in the projectile energy range between 150 and $1050 \mathrm{MeV} / \mathrm{u}[2]$. The comparison to microscopic calculations shows that nuclear matter is compressed to about two to three times the ground state density and that a substantial fraction of the kinetic energy in the entrance channel is converted into compressional energy [3]. In these calculations, the relation between density and compressional energy depends on the parameterization of the nucleon nucleon interaction. The values of its parameters will hopefully be restricted by a comparison of the calculations to experimental data, and thus one can obtain information on the 
(t) The members of the FOPI-collaboration:

J.P.Alard, Z.Basrak, N.Bastid, I.M.Belayev, M.Bini, R.Bock, A.Buta, R.Caplar, C.Cerruti, N.Cindro, J.P.Coffin, M.Crouau, P.Dupieux, J.Erö, Z.G.Fan, P.Fintz, Z.Fodor, R.Freifelder, L.Fraysse, S.Frolov, A.Gobbi, Y.Grigorian, G.Guillaume, N.Herrmann, K.D.Hildenbrand, S.Hölbling, O.Houari, S.C.Jeong, M.Jorio, F.Jundt, J.Kecskemeti, P.Koncz, Y.Korchagin, R.Kotte, M.Krämer, C.Kuhn, I.Legrand, A.Lebedev, C.Maguire, V.Manko, T.Matulewicz, G.Mgebrishvili, J.Mösner, D.Moisa, G.Montarou, P.Morel, W.Neubert, A.Olmi, G.Pasquali, D.Pelte, M.Petrovici, G.Poggi, F.Rami, W.Reisdorf, A.Sadchikov, D.Schül, Z.Seres, B.Sikora, V.Simion, S.Smolyankin, U.Sodan, N.Taccetti, K.Teh, R.Tezkratt, M.Trzaska, M.A.Vasiliev, P.Wagner, J.P.Wessels, T.Wienold, Z.Wilhelmi, D.Wohlfarth, A.V.Zhilin

\section{References}

[1] W.Scheid et al., Phys Rov Lett $\underline{32}, 741$ (1974)

[2] H.A.Gustafsson et al., Phys Rev Lett $\underline{52}, 1590$ (198.4)

[3] J.Aichelin, Phys Rep 202, 233 (1991)

B.Blättel et al., Phys Rev C43, 2728 (1991)

[4] J.Schambach et al., in: The Nuclear Equation of State, Proceedings of a NATO Advanced Study Institute, Peniscola, 1989, Vol. 216B, edited by W. Greiner and II. Stöcker, part A, p.115

[5] R.Madey et al., Procedings of the International Nuclear Physics Conference, Wiesbaden 1992, Nucl Phys 1553 , 779c (1993)

[6] A.Gobbi et al., Nucl Instr Meth 132t, 156 (1993)

[7] Th.Blaich et al., Nucl Instr Meth 1314, 136 (1992)

[8] Y.Leifels et al., Phys Rev Lett $\underline{71}, 963$ (1993)

[9] K.G.R.Doss et al., Phys Rev Lett $\underline{59}, 2720$ (1987)

[10] H.H.Gutbrod et al., Phys Rev C.12, 610 (1990)

[11] J.Aichelin and II.Stöcker, Phỵs Lett B176, 14 (1986)

[12] Ch.Hartnack, PhD thesis, Eniversity of l'rankfurt, 1992

[13] II.G.Ritter, Proceedings of this conference

[14] A.Bonasera et al., Nucl Phys $1.176,159$ (1988) and references therein 
nucleon-nucleon interaction within the nuclear medium which is not accessible from other sources.

This problem has until now mainly been attacked with data for charged particles. Such data are basically incomplete since the information on the neutrons is missing, and it is not a priori clear to what extent collective observables are modified by the long-range Coulomb interaction. It is therefore essential to compare neutrons and protons experimentally. At first sight, this seems to require the construction of a $4 \pi$ spectrometer for neutrons which is not feasible within reasonable cost. However, if the global observables of an event - like impact parameter or azimuthal angle of the reaction plane - are provided by a $\$ \pi$-spectrometer for charged particles, the spectrometer may be combined with a neutron detector of limited solid angle, and the combination will still deliver triple differential cross sections $d^{3} \sigma / d y d p_{t} d(\Delta \varphi)\left\{y\right.$ : rapidity, $\mathrm{p}_{t}$ : transverse momentum, $\Delta \varphi$ : azimuthal angle with respect to the reaction plane]. Thus the full information is obtained which is equivalent to a measurement in the complete solid angle.

Preliminary data from such an experiment have been reported earlier $[4,5]$. The neutron data were analyzed in terms of $\left\langle p_{\ell}\right\rangle$ in the reaction plane and azimuthal distributions about the beam axis. However, no analogous data for charged particles were taken, and hence the comparison to previous experiments is difficult. That difficulty is overcome by the combination of the FOPI-spectroneter [6] with the Large Area Neutron Detector LANI) [7]: The FOPI-spectrometer (phase I) covers the forward hemisphere of the cm-system for symmetric systems at energies above $\approx 150 \mathrm{MeV} / \mathrm{u}$ and provides the impact parameter vector. LAND determines the momentum vectors of the neutrons and light charged particles in a limited solid angle. They are thus detected in the same region of phase space, and their properties can be compared without any inter-or extrapolation, and without systematic errors due to different acceptances. The combination with $\mathrm{FOPI}$ allows for the exclusive investigation with respect to the impact parameter vector.

Our contribution focusses on one particular aspect of collective flow of nuclear matter: the so-called "squeeze-out", i.e. the preferential emission of mid-rapidity particles perpendicular to the reaction plane. The data were taken for the system ${ }^{197} \Lambda_{11}+{ }^{197} \mathrm{Au}$ at 400,600 and $800 \mathrm{MeV} / 1$. We will cover twe topics, the comparison of neutrons and protons, and the bombarding energy dependence of the neutrons' squeeze-out.

\section{EXPERIMENTAL SET-UP AND RESULTS}

The experimental set-up is shown schematically in fig. 1, and detailed descriptions of the two detector systems are given in refs. [6, 7]. Results from this experiment at a bombarding energy of $100 \mathrm{MeV} / \mathrm{u}$ have been published in [8]. The analysis at two higher energies, 600 and $800 \mathrm{McV} / \mathrm{u}$, was strictly analogous. Two global observables of each event, the azimuthal angle of the reaction plane and the impact parameter, were obtained from the charged particles in the plastic wall of the POPI spectrometer: (i) The azimuthal angle $\varphi_{R}$ of the reaction plane was taken from the total transverse momentum of the charged particles in the forward hemisphere. A flow tensor analysis was not performed, therefore all azimuthal angles are measured about the beam axis. Contrary to most other experiments, the problem of autocorrelations did not arise, since the particles to be correlated with the reaction plane those detected in IANI) were not involved in the determination of $\varphi_{R}$. (ii) The impact parameter was determined by the ratio of 'transwerse' to 'longitudinal' energy. For each event, $V_{1}=2 \frac{p_{\perp}^{2}}{2 m}$ and 


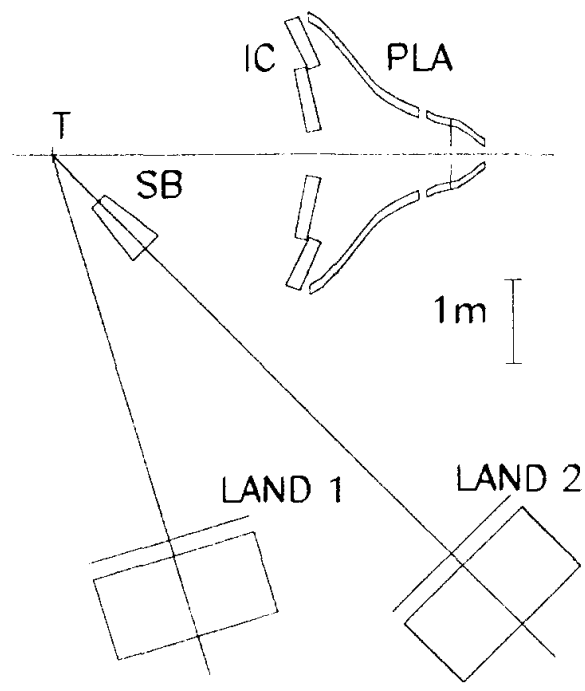

Figure 1: Set-up of the joint experiment of LAND) and the FOPI-spectrometer (phase I). The latter detects charged particles at $1^{\circ} \leq 0_{l a b} \leq 30^{\circ}$ with full coverage of the azimuthal angle. PLA indicates the internal and external plastic wall of scintillators, IC indicates a shell of ionization chambers for detection of heavy, slow clusters. For details, see ref. 6 . The two halves of $\mathrm{LAND}$ are positioned at $\theta_{l a b}=45^{\circ} \pm 8^{\circ}$ and $0_{l a b}=73^{\circ} \pm 12^{\circ}$, respectively. The figure gives as example the set-up for a shadow bar (SB) measurement with LA.NI) 2.

$E_{\|}=\Sigma \frac{p_{\|}^{2}}{2 m}$ were caiculated. 'The data were grouped into five bins of the 'energy ratio' $E_{\text {rat }}=E_{\perp} / E_{\|}$with bin names $E .1$ through E., with E.5 indicating the highest energy ratio. The transformation of $E_{\text {rat }}$ into absolute values of the impact parameter had to be done by means of model calculations. Since the data are to be compared to quantum molecular dynamics calculations (see below), the same model was chosen for the impact parameter transformation. Figure 2 shows the result for one particilar version, the so-called 'soft equation of state' with momentum-dependent interactions. As intuitively expected, the highest values of $E_{\text {rat }}$ are found to select the most central collisions. The correlation between $\mathrm{E}_{\text {rat }}$ and impact parameter depends only weakly on the QMD version used.

Since neutrons have a long range, their spectra contain background which originates from scattering from all massive objects within the experimental area and off the concrete walls. To investigate this effect, iron shadow bars were inserted between the target and each sub-system of LANI) separately, in such a way that the neutron detectors were shielded from particles coming directly from the target. During the analysis, all spectra were accumulated twice, once from runs without shadow bars and once from runs with the respective shadow bar in place. The spectra were then subtracted in order to eliminate the background. Table 1 shows the integral values in the two halves of $L A N D$ ) at the three projectile energies investigated. It is important to note that the background is event-correlated, i.e. some of the neutrons originate from the target, but reach the detector indirectly after scattering. 


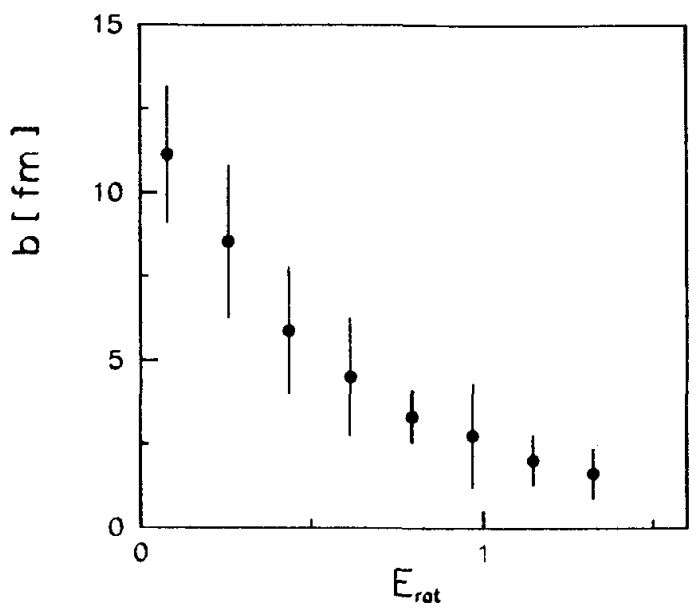

Figure 2: Relation between $E_{\text {rat }}$ and impact parameter from QMD calculations

\begin{tabular}{|c|c|c|}
\hline $\mathrm{E}_{\text {proj }}$ & LAND 1 & IAND 2 \\
\hline 100 & $10 \%$ & $30 \%$ \\
\hline 600 & $12 \%$ & $31 \%$ \\
\hline 800 & $1.1 \%$ & $33 \%$ \\
\hline
\end{tabular}

Table 1: Neutron background in the two halves of the LANl) detector

\section{SQUEEZE-OUT OF NEUTRONS AND PROTONS}

The squeeze-out effect is depicted in fig. 3 which shows typical data from the system ${ }^{197} \mathrm{Au}+{ }^{197} \mathrm{Au}$ at $600 \mathrm{MeV} / \mathrm{u}$. The figure shows azimuthal distributions about the beam axis of mid-rapidity neutrons (top) and hydrogen ions (bottom) for different impact parameter ranges which were selected by $\mathrm{E}_{\text {rat }}$. Only particles with rapidities $0.4 \leq \mathrm{y} / \mathrm{y}_{\text {proj }}<0.6$ are considered throughout the remainder of this contribution. 'The maxima of the count rate at $\Delta \varphi=\varphi-\varphi_{R}= \pm 90^{\circ}$ indicate the preferential emission perpendicular to the reaction plane. The anisotropy is very large at impact parameters which correspond to the radius of target and projectile, and it decreases with decreasing $b$ as the system becomes more and more azimuthally symmetric.

From a qualitative inspection of fig. 3 it is aiready obvious that the behaviour of neutrons and hydrogen ions is very similar. However, collective flow effects are known to increase with the mass of the particle considered, and therefore a separation of the hydrogen isotopes is essential. A scintillator wall in front of $\mathrm{LAN}$ ) yields the charge number of a particle from the correlation of energy loss with time of flight, whereas the mass number is obtained from calorimetric information from the neutron detector itself. Fig. 4 shows $\cdots$ for particles with $\%=1$ the ratio of the deposited energy and the kinetic energy per mass, which is given by the lorenty factor $\gamma-1$. One clearly sees a sharp peak at $0.13 \mathrm{GeV}$, a second peak at about twice that value, and a broad shoulder around $\mathrm{E}_{d e p} /(\gamma-1) \approx 0.4 \mathrm{GcV}$. It is obvious that the calorimetric resolution 


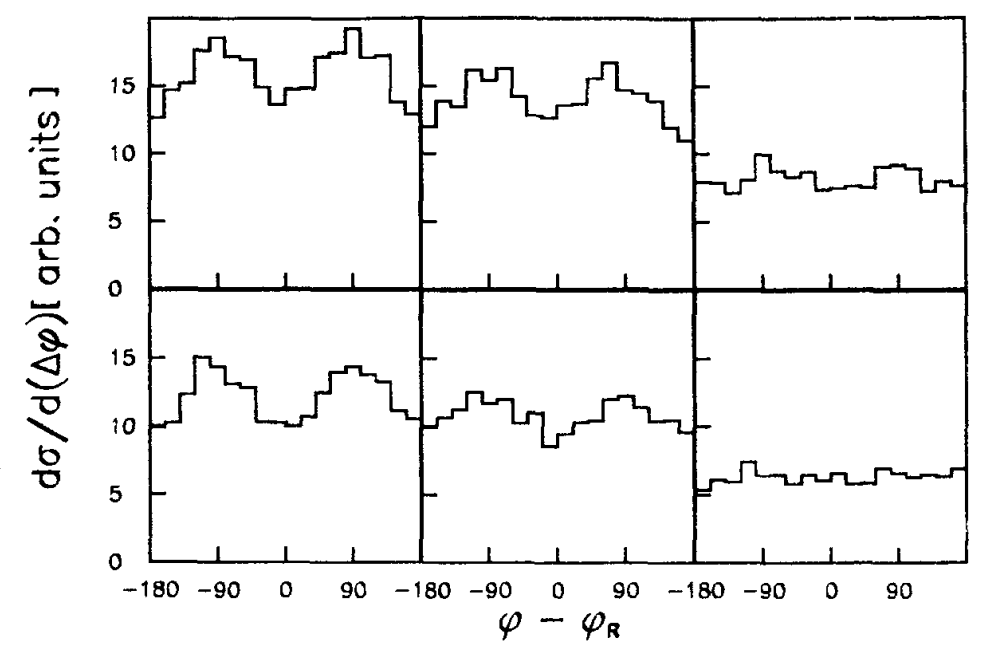

Figure 3: Azimuthal distributions of neutrons (top) and hydrogen ions (bottom) at impact parameters of $b=6.9 \pm 3.1 \mathrm{fm}$ (left), $b=4.2 \pm 1.8 \mathrm{fm}$ (middle), and $b=2.8 \pm 1.3 \mathrm{fm}$ (right).

is insufficient to separate the $\mathrm{H}$ isotopes event by event. However, we confirmed the correspondence of the peaks to the $\mathrm{H}$ isotopes by fitting the spectrum with three asymmetric peaks. Their positions and widths were fixed relative to each other at values of $1: 2: 3$ and $1: \sqrt{2}: \sqrt{3}$, respectively, and the asymmetry was described by only one parameter common to all three components. The resulting fit, which has only six free parameters, provides a good description of the spectrum as shown in the left part of fig. 4. Hence a cut on $\mathrm{E}_{d e p} /(\gamma-1)$ can be used to enrich $\mathrm{p}$, $\mathrm{d}$, and $\mathrm{t}$ in the azimuthal distributions and to get a feeling for the size of the signal as a function of mass. After applying very conservative cuts (right part of fig. 4), the azimuthal distributions were fitted with Legendre polynomials $\frac{d N}{d \Delta \varphi}=B_{0}+B_{1} \cdot P_{1}(x)+B_{2} \cdot P_{2}(x)$ with $\mathrm{x}=\cos (\Delta \varphi)$. The coefficient $\mathrm{B}_{2}$ describes the squeeze-out effect. Its values for mid-rapidity particles are shown in fig. 5. 'The results for neutrons and 'protons' are within the error bars the same, and the heavier isotopes show a larger anisotropy which is consistent with the earlier finding that collective effects show up more clearly in the data of heavier particles [9]. In addition, our results show that at relativistic energies collective observables are not affected by the Coulomb force despite its long range.

\section{ENERGY DEPENDENCE OF NEUTRON SQUEEZE-OUT}

We now turn to the dependence of the squecze-out signal on the bombarding energy. This investigation is done for the neutrons only, since in that instance we do not need to worry about isotope effects which are known to be substantial (sce above). The effect is summarized in fig. 6 which shows the squeeze-out ratio $R_{N}=\left\{N\left(+90^{\circ}\right)+\right.$ $\left.N\left(-90^{\circ}\right)\right\} /\left\{N\left(0^{\circ}\right)+N\left(180^{\circ}\right)\right\}$ as a function of the neut rons' perpendicular momentum. At the highest transverse momenta very high anisotropies are reached, higher than ever observed for baryons before. Fig. 6 (left part) shows that at all bombarding energies 


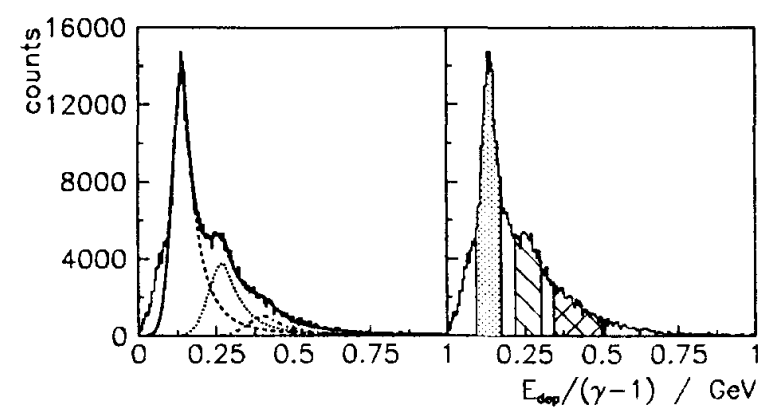

Figure 4: Enrichment of hydrogen ions by means of the energy deposited in the LAND detector. For details, see text.

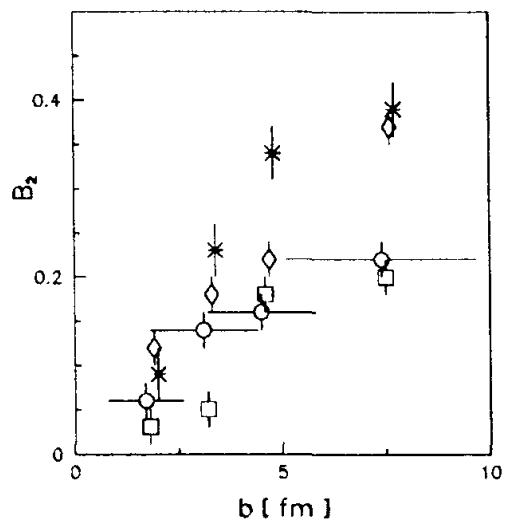

Figure 5: Coefficients of $2^{\text {nd }}$-order legendre polynomials for neutrons (circles) and particles in the p-cut of fig. 4 (squares), in the d-cut (diamonds), and in the t-cut(asterisks).

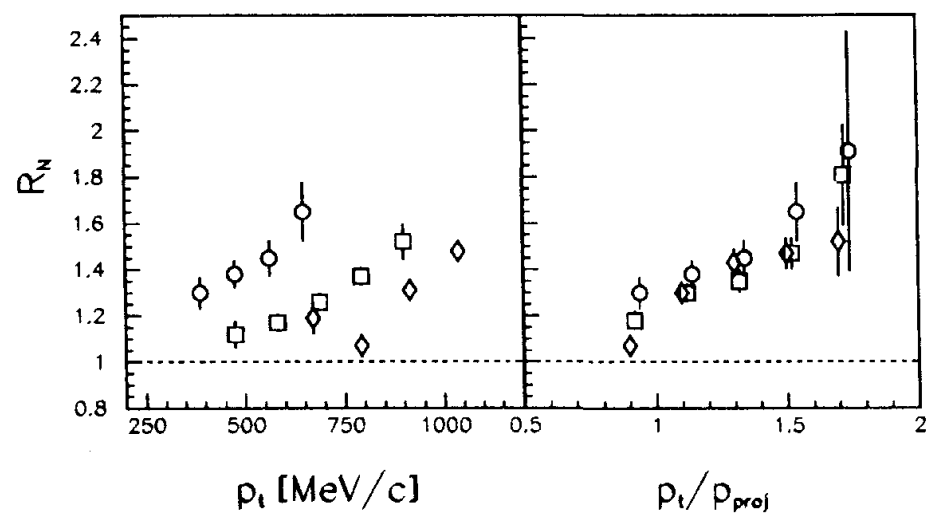

Figure 6: Squeeze-out ratio for neutrons as a function of perpendicular momentum at $\mathrm{E}_{\text {proj }}=100 \mathrm{MeV} / \mathrm{u}$ (circles), $600 \mathrm{MeV} / \mathrm{u}$ (squares), and $800 \mathrm{McV} / \mathrm{u}$ (diamonds). An $E_{\text {rat }}$-cut was applied which selects impact parameters of about $7 \pm 3 \mathrm{fm}$. 


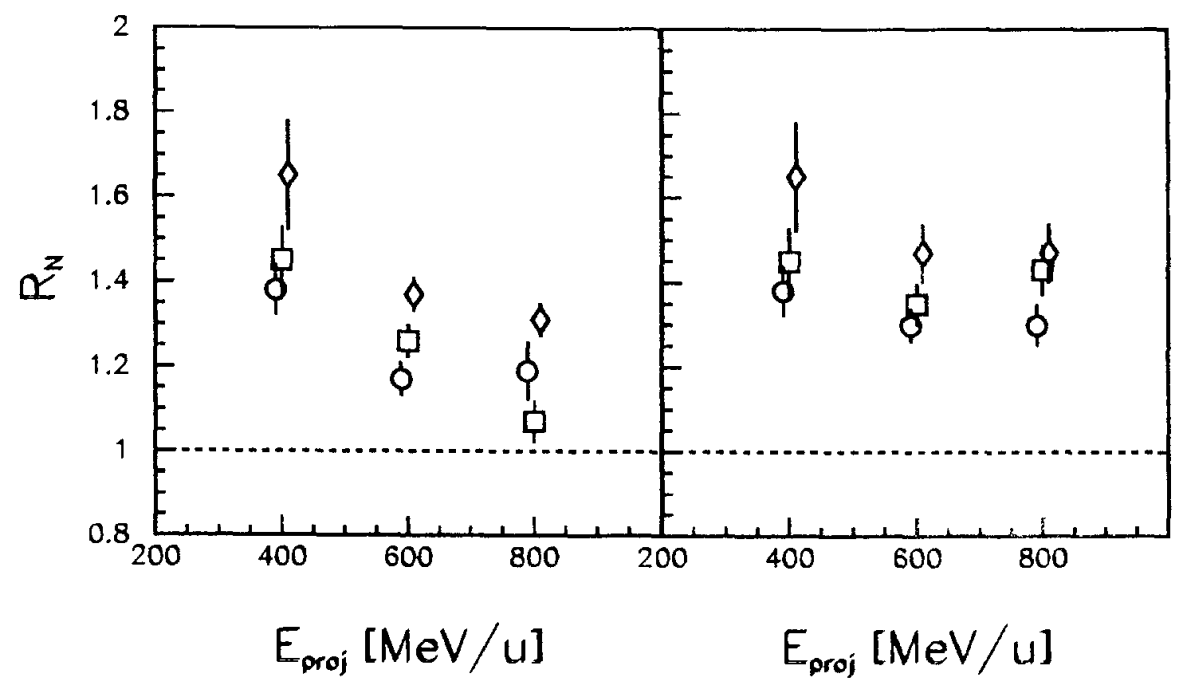

Figure 7: Squeeze-out ratio for neutrons as a function of bombarding energy. An $E_{\text {rat }}$-cut was applied which selects impact parameters of about $7 \pm 3 \mathrm{fm}$. The left part shows $R_{N}$ for neutrons with $p_{t}=390 \mathrm{MeV} / \mathrm{c}$ (circles), $\mathrm{p}_{t}=470 \mathrm{MeV} / \mathrm{c}$ (squares), and $\mathrm{p}_{t}=550 \mathrm{MeV} / \mathrm{c}$ (diamonds) in bins of $80 \mathrm{MeV} / \mathrm{c}$. The right part shows $\mathrm{R}_{N}$ for neutrons with $\mathrm{p}_{t}=0.9 \cdot \mathrm{p}_{\text {proj }}, \mathrm{p}_{t}=1.1 \cdot \mathrm{p}_{\mathrm{proj}}$, and $\mathrm{p}_{t}=1.3 \cdot \mathrm{p}_{\mathrm{proj}}$, respectively.

the maximum values of $R_{N}$ are the same, but they are reached at different transverse momenta: With increasing energy, the squecze-out signal is shifted to increasingly higher $p_{t}$. The effect becomes even more striking if the transverse momentum of the neutrons is not measured in absolute numbers, but in fractions of the projectile mo mentum: The dependence of $R_{N}$ on $p_{t}$ leads to a universal curve, independent of bombarding energy, as shown in the right part of fig. 6 . We are thus led to a new interpretation of the excitation function: The latter has been shown to decrease with energy, if no cuts on $\mathrm{p}_{t}$ are applied (fig. 12 from [10]). This situation corresponds to an acceptance cut on $p_{t}$ in absolute values, and the result is reproduced if more detailed cuts are applied (left part of fig. 7). However, if the cut on $p_{t}$ is implemented in units of projectile momentum, the excitation function of $R_{N}$ becomes flat (right part of fig. 7 ).

These findings remind one of the scaling laws of non-viscous fluid dynamiss. We need to point out, however, that the effect is clearly observed in a fairly narrow impact parameter range where the squecze-out signal is large, but the signature gets less clear if we go to smaller $b$, although indications of the effect remain.

\section{COMPARISON TO THEORY}

In an attempt to interpret our data quantitatively, we compared them to microscopic calculations in the framework of quantum molecular dynamics (Q.MI)) [11]. Since we are discussing the behaviour of neutrons, we chose the recently developed version of IQMI) [12] which kecps track of isotopic spin. We restricted ourselves to two versions which both contain momentum dependent interactions, but use different compressibilities of infinite nuclear matter ('hard' and 'soft' cquation of state). 'The 


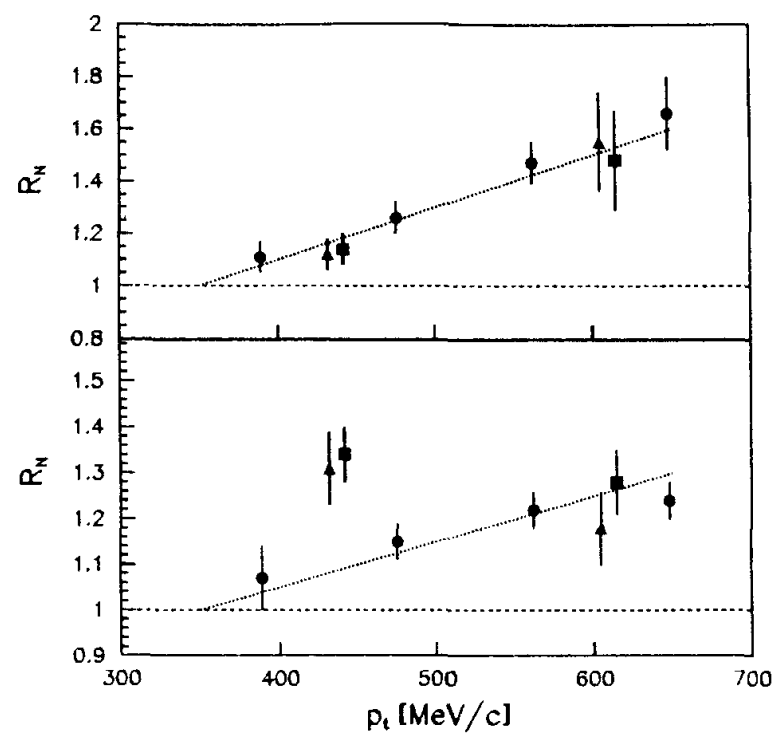

Figure 8: Squeeze-out ratio for neutrons as a function of perpendicular momentum at $\mathrm{E}_{\text {proj }}=400 \mathrm{MeV} / \mathrm{u}$ (top) and $600 \mathrm{MeV} / \mathrm{u}$ (bottom). An $\mathrm{E}_{\text {rat }}$-cut was applied which selects impact parameters of about $7 \pm 3 \mathrm{fm}$. Circles: experimental data, squares: QMD results with hard EOS, triangles: QMD results with soft EOS.

results of the calculations were filtered event-wise by the acceptance of the IAND- and IOPI-detectors, and then treated exactly like data: $E_{\text {rat }}$ was used to cut on the impact parameter, the total transverse momentum was used to re-determine the reaction plane, and azimuthal distributions of neutrons were analyzed strictly analogous to the experimental data. The result of the comparison is shown in fig. 8: $A \mathrm{t} 400 \mathrm{MeV} / \mathrm{u}$, both versions of IQMD fit the data very nicely, however, at $600 \mathrm{MeV}$, the calculations are not able to reproduce the $\mathrm{p}_{t}$-dependency of the squeeze-out signal. The experimental values are rising with $p_{t}$, whereas the calculated values show the opposite behaviour. As of this writing, the reason is unclear, and detailed investigations of the model are under way.

Until now, we have been dealing with squecze-out which is related to collective flow perpendicular to the reaction plane. We have shown that in a certain impact parameter range, the squecze-out signal scales with projectile momentum. Naturally, the question arises whether a similar behaviour can be found in other observables pertaining to collective effects. In particular, it is interesting to look at the 'flow parameter' $F$, i.e. the slope of the $\left\langle p_{t}\right\rangle$ y curve at $y=y_{\mathrm{cm}} .\left\langle\mathrm{p}_{t}\right\rangle$ is the transverse momentum in the reaction plane, averaged over the particles of the event. At a given bombarding energy, $\mathrm{F}$ goes through a maximum if plotted versus impact parameter. A preliminary excitation function of the maximum $\mathrm{l}_{\text {max }}$ for the system ${ }^{197} \mathrm{Au}+{ }^{197} \mathrm{Au}$ has been presented by the EOS collaboration [13]. It is rising continuously if $\left\langle p_{t}\right\rangle$ is measured in absolute values, whereas scaling of $\left\langle p_{\ell}\right\rangle$ with $p_{p r n j}$ yields a value of $\approx 30 \%$ independent of energy (see fig. 9). 'lhus the flow of $\left\langle p_{t}\right\rangle$ in the reaction plane exhibits the same scaling behaviour as the flow out of the reaction plane. This kind of scaling has been investigated earlier for the in-plane flow ([14]) in the frame work of hydrodynamical models, but our observation of this phenomenon for the out-of-plane flow calls for more quantitative calculations. 


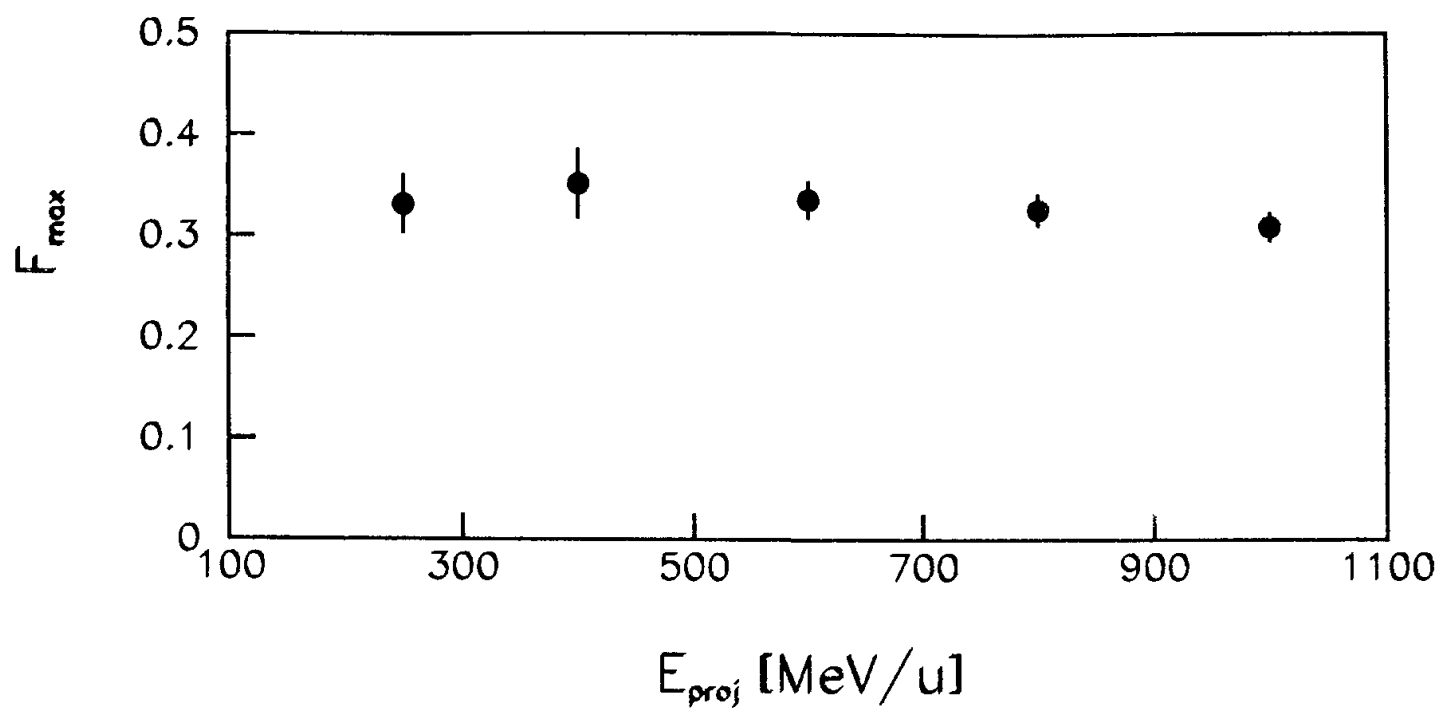

Figure 9: Maximum flow parameter versus bombarding energy for the system ${ }^{197} \mathrm{Au}+$ ${ }^{197} \mathrm{Au}$. For details, see text.

\section{SUMMARY}

In summary, we have presented a detailed investigation of the squeeze-out effect for the ${ }^{197} \mathrm{Au}+{ }^{197} \mathrm{Au}$ system in the energy range $400 \mathrm{NeV} / \mathrm{u} \leq \mathrm{F}_{\text {proj }} \leq 800 \mathrm{MeV} / \mathrm{u}$. We have shown that the effect is the same for neutrons and protons, which means that the Coulomb force - despite its long range - does not have a significant effect on the observables of collective flow. The size of the squeeze-out signal depends on the transverse momentum of the particles observed. The $p_{t}$-dependence scales with the momentum of the projectile such that a plot of $\mathrm{R}_{N}$ versus $\mathrm{p}_{t} / \mathrm{p}_{\text {proj }}$ leads to a universal curve, at least for large impact parameters where the signal is large. This scaling behaviour for out-of-plane momentum flow is also found for in-plane moment $1 \mathrm{~m}$ flow as measured by the slope of the $\left\langle\mathrm{p}_{t}\right\rangle-\mathrm{y}$ curve at $\mathrm{y}=y_{\mathrm{cm}}$, and detailed comparisons to microscopic as well as macroscopic calculations seem necessary for an understanding of this effect.

Acknowledgements:

We thank the accelerator personnel at GSI for providing an excellent ${ }^{197}{ }^{\prime} u$-beam for our experiment. This work was funded by the German liederal Secretary for Research and 'lechnology (BMF'I) under contract numbers (06) BO 103, 06 OF 112, and $06 \mathrm{MZ}$ 106, and by GSI via Hochschulzusammenarbeitsvereinbarungen under contract numbers BO FRE, F ELE, and VZ KRI). This work was partly supported by the Committee for Scientific Research (Poland) under grant Vo. P131158/P3/92. 